\title{
BRANCH POINT STRUCTURE OF COVERING MAPS ONTO NONORIENTABLE SURFACES
}

\author{
BY \\ CLOYD L. EZELL 1
}

ABSTRACT. Let $f: M \rightarrow N$ be a degree $n$ branched cover onto a compact, connected nonorientable surface with branch points $y_{1}, y_{2}, \ldots, y_{m}$ in $N$, and let the multiplicities at points in $f^{-1}\left(y_{i}\right)$ be $\mu_{i 1}, \mu_{i 2}, \ldots, \mu_{i k_{i}}$. The branching array of $f$, designated by $B$, is the following array of numbers:

$$
\begin{gathered}
\mu_{11}, \mu_{12}, \ldots, \mu_{1 k_{1}} \\
\mu_{21}, \mu_{22}, \ldots, \mu_{2 k_{2}} \\
\vdots \\
\mu_{m 1}, \mu_{m 2}, \ldots, \mu_{m k_{m}}
\end{gathered}
$$

We show that the numbers in the branching array must always satisfy the following conditions:

(1) $\Sigma\left\{\mu_{i j}+1 \mid j=1,2, \ldots, k_{i}\right\}=n$,

(2) $\Sigma\left\{\mu_{i j} \mid i=1,2, \ldots, m ; j=1,2, \ldots, k_{i}\right\}$ is even.

Furthermore, if $B$ is any array of numbers satisfying these conditions, and if $N$ is not the projective plane, then there is a branched cover onto $N$ with $B$ as its branching array.

1. Introduction. Let $f: M \rightarrow N$ be a continuous map between 2-dimensional manifolds. An open set $U \subset N$ is evenly covered if $f^{-1}(U)$ is a union of disjoint open sets on each of which $f$ is topologically equivalent to the complex map $z^{n}$, for some $n$. If every point $y \in N$ has an evenly covered neighborhood, then $f$ is called a branched cover and the pair $(f, M)$ a branched covering space of $N$. If $f$ is equivalent to $z^{n}$ at $x$, then the local degree of $f$ at $x$ is $n$ and the multiplicity of $x$ is $n-1$. A point $x \in M$ with positive multiplicity is a critical point and a point $y \in N$ with at least one preimage a critical point is a branch point. If $y$ and $z$ in $N$ are not branch points, then $\left|f^{-1}(y)\right|$ (the cardinality of $\left.f^{-1}(y)\right)$ equals $\left|f^{-1}(z)\right|$ and we may define the degree of $f$, $\operatorname{deg} f$, to be $\left|f^{-1}(y)\right|$, for any $y$ not a branch point. Two branched covers $f_{i}$ :

Presented to the Society, November 17, 1976; received by the editors December 10, 1976 and, in revised form, July 23, 1977.

AMS (MOS) subject classifications (1970). Primary 55A10; Secondary 30A90.

Key words and phrases. Branched cover, branching array, multiplicity of a branched cover, nonorientable surface.

'The results presented in this paper form a part of the author's $\mathrm{Ph}$. D thesis, written at Vanderbilt University under the direction of Professor Morris L. Marx.

- American Mathematical Society 1978 
$M_{i} \rightarrow N, i=1,2$, are equivalent if there exists a homeomorphism $h: M_{1} \rightarrow M_{2}$ such that $f_{1}=f_{2} h$.

In this paper we will be concerned with branched covers between compact, closed (no boundary), connected surfaces, either orientable or nonorientable. In this case the number of branch points, the number of critical points, and the degree are all finite. Let $f: M \rightarrow N$ be such a map with branch point set $Y=\left\{y_{1}, y_{2}, \ldots, y_{m}\right\}$ in $N$ and let $\mu_{i 1}, \mu_{i 2}, \ldots, \mu_{i k_{i}}, i=1, \ldots, m$, be the multiplicities of the preimages of $y_{i}$. The branching array of $f$, designated by $B$, is the following array of numbers:

$$
B=\left[\begin{array}{c}
\mu_{11}, \mu_{12}, \ldots, \mu_{1 k_{1}} \\
\mu_{21}, \mu_{22}, \ldots, \mu_{2 k_{2}} \\
\vdots \\
\mu_{m 1}, \mu_{m 2}, \ldots, \mu_{m k_{m}}
\end{array}\right]
$$

We say an array of numbers $B$ is admissible if there is a branched cover having $B$ as its branching array. The sum, designated by $\mu$, of all of the entries in the branching array of some map is called the multiplicity of the map. A well-known fact concerning the multiplicities of branched covers is the Riemann-Hurwitz formula; namely, if $f: M \rightarrow N$ is a degree $n$ branched cover with multiplicity $\mu$, then $\mu=n \cdot \chi(N)-\chi(M)$, where $\chi$ represents the Euler characteristic of a surface.

Many of the problems concerning branching arrays of branched covers fall into one of two categories: enumeration problems and branch point classification problems. In an enumeration problem, one considers a class of maps $F$ and an array $B$ which is admissible for some map in $F$. The problem is to count the number of topologically distinct maps in $F$ with branching array $B$. In a classification problem, one considers the set $\mathscr{B}$ of all branching arrays corresponding to maps in some class $F$. The problem is to find necessary and sufficient conditions for an array of numbers $B$ to be a member of $B$.

An example of an enumeration problem appears in the work of Hurwitz [4]. Hurwitz enumerated all $n$-sheeted Riemann surfaces over the sphere with $2 n-2$ simple branch points. Translating to our language, Hurwitz enumerated all degree $n$ branched covers over the sphere having the branching array:

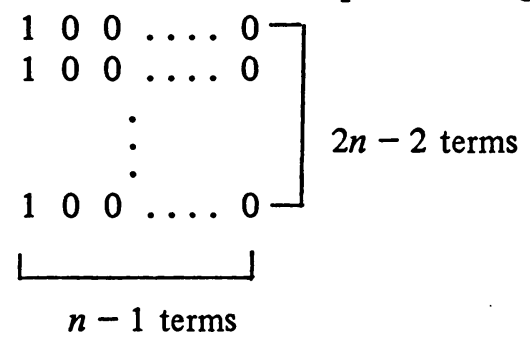


Note that it follows from the Riemann-Hurwitz formula that the covering surface must also be the sphere.

Examples of classification problems appear in papers by Thom [7] and Shepardson [6]. In Thom's problem, the class of maps were the degree $n$ polynomials $p: S^{2} \rightarrow S^{2}$, where $S^{2}$ is the Riemann sphere. If $B=\left\{\mu_{i j} \mid i=\right.$ $\left.1, \ldots, m ; j=1, \ldots, k_{i}\right\}$ is the branching array of a degree $n$ polynomial, then the following conditions must be satisfied:

(1) $k_{i}=1$, for some $i$ ( $\infty$ is a branch point with one preimage);

(2) $\Sigma\left\{\mu_{i j}+1 \mid j=1, \ldots, k_{i}\right\}=n$;

(3) $\mu=2 n-2$.

Thom showed that any array of numbers satisfying (1)-(3) is the branching array of some degree $n$ polynomial.

In Shepardson's problem, the maps were the degree $n$ branched covers onto an arbitrary orientable surface $N$ with $\chi(N)<0$ (i.e. $N$ can be any orientable surface except the sphere). Shepardson showed that the branching arrays corresponding to members in this class are characterized by the conditions:

(1) $\Sigma\left\{\mu_{i j}+1 \mid j=1, \ldots, k_{i}\right\}=n$;

(2) $\mu$ is an even number.

In this paper we extend Shepardson's result to include the nonorientable surfaces; i.e., we show that the above two conditions characterize the branching arrays of degree $n$ branched covers onto any surface $N$, orientable or nonorientable, as long as $\chi(N) \leqslant 0$.

The branching arrays for maps onto the sphere and projective plane have not been characterized, although some observations on certain admissible arrays for maps onto the sphere have been made by Endler [1]. Also, Francis [2] has defined a combinatorial invariant, called an assemblage, with which one can determine certain admissible arrays for maps from bordered surfaces into the sphere.

The facts on branched covers that we use to obtain these results are known but not readily available in the literature. Hence a summary of these is given in $\$ 2$ of this paper. The actual classification problem is done in $\$ 3$.

2. Representation of branched covers. Let $f: M \rightarrow N$ be a degree $n$ branched cover between compact, closed surfaces with $N$ connected. ( $M$ is not necessarily connected.) Let $Y=\left\{y_{1}, y_{2}, \ldots, y_{m}\right\}$ be a set of points in $N$ containing the branch points, $\bar{N}$ the punctured surface $\bar{N}=N-Y$, and $\Pi(\bar{N}, x)=\Pi$ the fundamental group of $\bar{N}$ based at some point $x \in \bar{N}$. The map $f$ induces a homomorphism $\rho: \Pi \rightarrow S_{n}$, where $S_{n}$ is the symmetric group of permutations on the letters $\{1,2, \ldots, n\}$, in the following way. Let the preimages of $x$ be $x_{1}, \ldots, x_{n}$. For each element $\omega \in \Pi$, define $\rho(\omega)=\rho_{\omega}$ as follows: $\rho_{\omega}(i)=j$ if the lift of some path in $\omega$ to $x_{i}$ terminates at $x_{j}$. Since 
each such path in $\omega$ terminates at the same point, $\rho_{\omega}$ is well defined, and since there are no branch points in $\bar{N}, \rho_{\omega}$ is one-one and a permutation in $S_{n}$. The map $\rho$ is easily seen to be a homomorphism.

The homomorphism $\rho$ determined by $f$ is not unique. In fact $f$ determines an entire equivalence class of homomorphisms as follows. Define homomorphisms $\rho$ and $\delta$ to be equivalent if there exists a permutation $\sigma \in S_{n}$ such that for every $\omega \in \Pi, \rho_{\omega}=\sigma^{-1} \delta_{\omega} \sigma$. Now if $f$ induces $\rho$ and if $\rho$ is related to $\delta$, a relabeling of the subscripts of $f^{-1}(x)$ by $\sigma$ causes $f$ to induce $\delta$. Conversely, any relabeling of the subscripts causes $f$ to induce another homomorphism related to $\rho$. We now observe that two branched covers which determine the same class of homomorphisms are themselves equivalent.

Let $f: M \rightarrow N$ and $g: L \rightarrow N$ be degree $n$ branched covers with branch points in the set $Y=\left\{y_{1}, \ldots, y_{m}\right\}$ and suppose $f$ and $g$ induce equivalent homomorphisms $\rho$ and $\delta$, respectively. Let $x_{1}, \ldots, x_{n}$ and $z_{1}, \ldots, z_{n}$ be the preimages of $x$ in $M$ and $L$ and suppose $x_{1}, \ldots, x_{n_{1}}$ are the preimages of $x$ in some connected component of $M$, say $M_{1}$. Then the existence of $\sigma$ implies that $z_{\sigma(1)}, \ldots, z_{\sigma\left(n_{1}\right)}$ are the preimages of $x$ in some component of $L$, say $L_{1}$. We now observe that the covering spaces $\left(\bar{f}_{1}, \bar{M}_{1}\right)$ and $\left(\bar{g}_{1}, \bar{L}_{1}\right)$ of $\bar{N}$ (where $\bar{M}$ and $\bar{L}$ are the punctured surfaces obtained by removing the $x_{i}$ 's and $z_{i}$ 's, and $\bar{f}_{1}$ and $\bar{g}_{1}$ are the restrictions of $f$ and $g$ to these surfaces) are equivalent.

If $\bar{f}_{1}^{\#}: \Pi\left(\bar{M}_{1}, x_{1}\right) \rightarrow \Pi$ and $\bar{g}_{1}^{\#}: \Pi\left(\bar{L}_{1}, z_{\sigma(1)}\right) \rightarrow \Pi$ are the usual monomorphisms from the fundamental groups of the covering spaces (see [5, p. 154]), then we have

$$
\begin{aligned}
\bar{f}_{1}^{\#}\left(\Pi\left(\bar{M}_{1}, x_{1}\right)\right) & =\left\{\omega \in \Pi \mid \rho_{\omega}(1)=1\right\}=\left\{\omega \in \Pi \mid \sigma^{-1} \delta_{\omega} \sigma(1)=1\right\} \\
& =\left\{\omega \in \Pi \mid \delta_{\omega}(\sigma(1))=\sigma(1)\right\}=\bar{g}_{1}^{\#}\left(\Pi\left(\bar{L}_{1}, z_{\sigma(1)}\right)\right) .
\end{aligned}
$$

Hence the covering spaces are equivalent (see [5, p. 159]), and by replacing the excised points, we have that the branched covers $f_{1}$ and $g_{1}$ (the restrictions of $f$ and $g$ to $M_{1}$ and $L_{1}$ ) are equivalent. Similarly, one can show that there is a one-one correspondence between the components of $M$ and $L$ such that the restrictions of $f$ and $g$ to corresponding components are equivalent branched covers.

Conversely, it is easily shown that equivalent branched covers determine the same equivalence class of homomorphisms.

If we let $\mathscr{F}$ be the set of all equivalence classes of degree $n$ branched covers over $N$ with branch set in $Y$, and $\mathcal{P}$ the set of equivalence classes of homomorphisms $\rho: \Pi \rightarrow S_{n}$, then we have established a one-one map $\Gamma$ from $\mathscr{F}$ to $\mathcal{P}$. We will now observe, using some construction techniques of Hurwitz, that this map is actually onto all of $\mathcal{P}$.

Now suppose we are given a closed, connected surface $N$, a finite set of 
points $Y=\left\{y_{1}, \ldots, y_{m}\right\}$ in $N$, and a homomorphism $\rho: \Pi \rightarrow S_{n}$. We can construct a degree $n$ branched cover $f: M \rightarrow N$, from some surface $M$, which induces $\rho$ as described below.

Consider a system of generators of $\Pi$. For convenience, we choose the generators in the standard way; namely, $\Pi$ is generated by $\alpha_{1}, \ldots, \alpha_{r}, \beta_{1}, \ldots, \beta_{m}$, where the $\alpha_{i}$ 's are generators of $\Pi(N, x)$ and the $\beta_{i}$ 's are path classes represented by simple closed curves about the points $y_{i}$. Then $\Pi$ is represented by these generators with the single relation $W(\beta) W(\alpha)=1$ (the identity in II), where $W(\beta)$ is the word $\beta_{1} \beta_{2} \ldots \beta_{m}$ and $W(\alpha)$ is the word $\alpha_{1} \alpha_{2} \alpha_{1}^{-1} \alpha_{2}^{-1} \ldots \alpha_{r-1} \alpha_{r} \alpha_{r-1}^{-1} \alpha_{r}^{-1}, r=2 p$, for $N$ orientable of genus $p$, and $\alpha_{1}^{2} \alpha_{2}^{2} \ldots \alpha_{r}^{2}, r=p$, for $N$ nonorientable of genus $p$.

Let $a_{1}, \ldots, a_{r}$ be closed curves intersecting only at $x$ representing the path classes $\alpha_{1}, \ldots, \alpha_{r}$, and let $b_{1}, \ldots, b_{m}$ be a set of nonintersecting line segments connecting $x$ and the points $y_{i}$. See below.

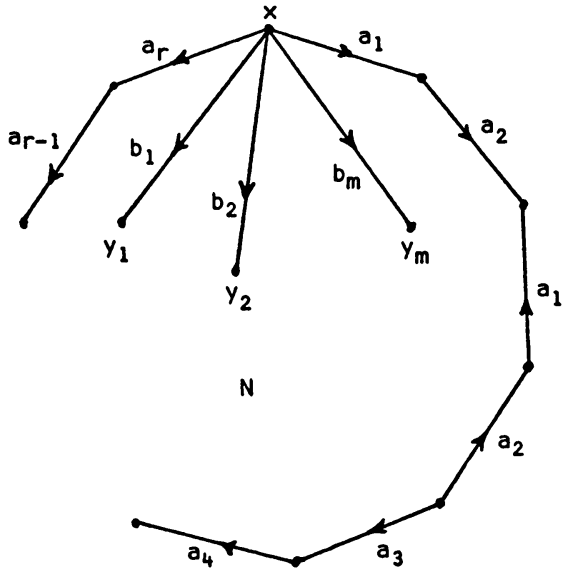

$N$ orientable

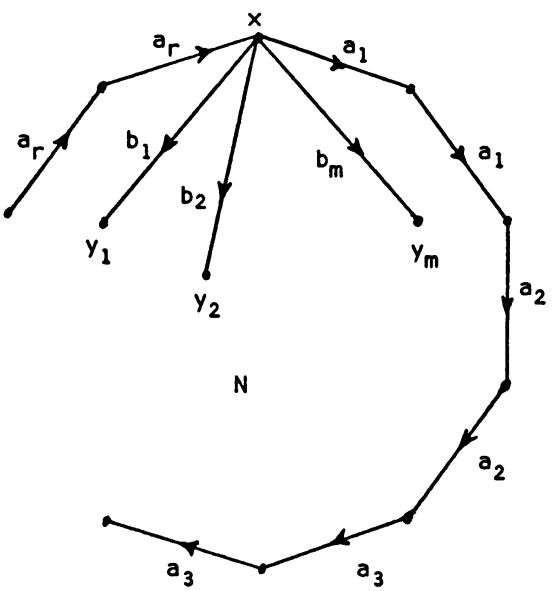

$\mathrm{N}$ nonorientable

FIGURE 1

Decompose $N$ by cutting along the curves $a_{i}$ and the lines $b_{i}$ to obtain a closed disk $Z$ with oriented boundary edges corresponding to the letters in the word $W(\beta) W(\alpha)$; i.e., $b_{1} b_{1}^{\prime} \ldots b_{m} b_{m}^{\prime} a_{1} a_{2} a_{1}^{\prime} a_{2}^{\prime} \ldots a_{r-1} a_{r} a_{r-1}^{\prime} a_{r}^{\prime}$ for $N$ orientable and $b_{1} b_{1}^{\prime} \cdots b_{m} b_{m}^{\prime} a_{1} a_{1}^{\prime} a_{2} a_{2}^{\prime} \cdots a_{r} a_{r}^{\prime}$ for $N$ nonorientable. See below.

Construct the covering surface $M$ by taking $n$ copies of $Z, Z_{1}, \ldots, Z_{n}$, and identifying the oriented edges as follows. If $w$ (either an $a_{i}$ or $b_{i}$ ) is an edge on the boundary of $Z$ for which $\rho(\omega)$ (where $\omega$ is the corresponding $\alpha_{i}$ or $\beta_{i}$ ) in $S_{n}$ maps $k$ to $j$, attach $Z_{k}$ to $Z_{j}$ by identifying edges $w^{k}$ and $\left(w^{j}\right)^{\prime}$. (The superscripts indicate the disk containing the edge.) Define a degree $n$ branched cover $f: M \rightarrow N$ by taking homeomorphisms $f_{k}: Z_{k} \rightarrow Z, k=$ $1, \ldots, n$, and extending in the obvious way. One can label the preimages of $x$ 


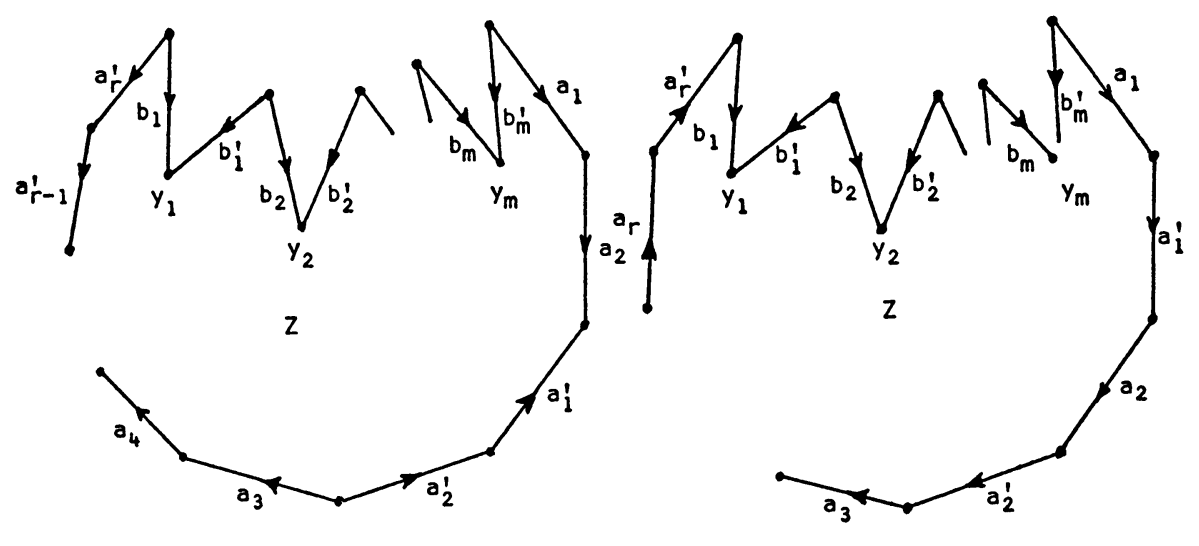

$\mathrm{N}$ orientable

$N$ nonorientable

\section{Figure 2}

so that $f$ does induce the homomorphism $\rho$ from which it was constructed.

By the construction, it is clear that the only possible branch points are the $y_{i}$ and $x$. The multiplicities of the preimages of $y_{i}$ are determined by the cycles in the permutation $\rho\left(\beta_{i}\right)$ as follows. If $\rho\left(\beta_{i}\right)$ has a cycle $\left(c_{1} c_{2} \cdots c_{t}\right)$, then by the construction, the oriented boundary of a small disk about $y_{i}$ lifts to arcs in sheets $c_{1}, c_{2}, \ldots, c_{t}$, respectively. Hence $y_{i}$ has a preimage at which $f$ is locally $t$-to-one. Thus if $\rho\left(\beta_{i}\right)$ has $k_{i}$ cycles of lengths $\mu_{i 1}+1, \ldots, \mu_{i k_{1}}+1$, then $y_{i}$ has $k_{i}$ preimages with multiplicities $\mu_{i 1}, \ldots, \mu_{i k_{i}}$. Since $\rho$ is a homomorphism, $W(\rho(\beta)) W(\rho(\alpha))=1$ (the identity in $S_{n}$ ), and it follows that $x$ has $n$ preimages and cannot be a branch point. Hence all branch points are in $Y$.

If the target surface $N$ is orientable, then it is well-known that any covering surface must be orientable. (Note that this is readily checked from the construction above.) For nonorientable targets, the orientability of the covering surface can be determined by examination of the generators of $\Pi$ as described below.

Suppose $N$ is nonorientable. A curve in $M$ avoiding the preimages of $x$ and crossing the lifts of the $a_{i}$ 's and $b_{j}$ 's transversely is orientation reversing if and only if it crosses the lifts of the $a_{i}$ 's an odd number of times. Hence $M$ is nonorientable if and only if there exists such a curve in $M$. Suppose $W(\alpha, \beta)$ $=\omega_{1} \omega_{2} \cdots \omega_{q}$ is a word in $\Pi$ with an odd number of occurrences of $\alpha_{i}^{\prime}$ 's and suppose the permutation $W(\rho(\alpha), \rho(\beta)$ ) (i.e. the permutation $\rho\left(\omega_{q}\right) \cdots \rho\left(\omega_{2}\right) \rho\left(\omega_{1}\right)$, multiplying from right to left) fixes some number $k \in$ $\{1, \ldots, n\}$. Let $w_{i}, i=1, \ldots, q$, be the edges in $N$ corresponding to the $\omega_{i}$ 's. ( $\omega_{i}$ is either $\alpha_{i}^{ \pm 1}$ or $\beta_{i}^{ \pm 1}$ and $w_{i}$ is the corresponding $a_{i}$ or $b_{i}$ ) Construct a path by beginning at some point $z \in Z_{k}$, cross edge $w_{1}^{k}$ to sheet $Z_{\rho\left(\omega_{1}\right)(k)}$, cross $w_{2}^{\rho\left(\omega_{1}\right)(k)}$ to sheet $Z_{\rho\left(\omega_{2}\right) \rho\left(\omega_{1}\right)(k)}$, etc. Since $\rho\left(\omega_{q}\right) \cdots \rho\left(\omega_{2}\right) \rho\left(\omega_{1}\right)(k)=k$, this 
process produces a path ending at the original sheet $Z_{k}$. Terminate the path at the starting point $z \in Z_{k}$. Since there are an odd number of $\alpha$ terms in $W(\alpha, \beta)$, we cross the edges $a_{i}$ an odd number of times, and the path is reversing. Similarly, the existence of such a path implies the existence of a word $W(\alpha, \beta)$ with an odd number of $\alpha$ terms such that $W(\rho(\alpha), \rho(\beta))$ fixes some letter $k$.

Finally, we note that if $\rho(\mathrm{II})$ acts transitively on $\{1, \ldots, n\}$, then we can construct a path in $M$ from $Z_{k}$ to $Z_{j}$, for any choice of $k$ and $j$, and thus $M$ must be connected. Conversely, the existence of such a path implies $\rho(\mathrm{II})$ acts transitively. More generally, if we let $i$ be related to $j$ if there exists a permutation $P \in \rho(I I)$ such that $P(i)=j$, and if $S_{1}, \ldots, S_{k}$ are the equivalence classes which partition the set $\{1, \ldots, n\}$ under this relation, then $M$ has $k$ connected components and the restrictions of $f, f_{1}, \ldots, f_{k}$, to these components have degrees $\left|S_{1}\right|, \ldots,\left|S_{k}\right|$.

We summarize all of the above in the following theorem. As before, $N$ is some connected, closed surface, $Y=\left\{y_{1}, \ldots, y_{m}\right\}$ are selected points in $N$, $\bar{N}=N-Y, \mathcal{F}$ is the set of all equivalence classes of degree $n$ branched covering spaces of $N$ with branch point set in $Y$, and $\mathcal{P}$ is the set of all equivalence classes of homomorphisms $\rho: \Pi \rightarrow S_{n}$. We assume $\Pi$ is represented in the standard way as described earlier.

2.1. THEOREM. There is a one-one correspondence $\Gamma: \mathcal{F} \rightarrow \mathcal{P}$ such that if $\Gamma(F)=H,(f, M) \in F$, and $\rho \in H$, then:

(1) There is a one-one correspondence between the connected components of $M, M_{1}, \ldots, M_{k}$ and the equivalence classes $S_{1}, \ldots, S_{k}$ of $\{1, \ldots, n\}$ determined by the action of $\rho(\Pi)$ on $\{1, \ldots, n\}$ such that the degree of $f$ restricted to $M_{i}$ equals $\left|S_{i}\right|$. Specifically, $M$ is connected if and only if $\rho(\mathrm{II})$ acts transitively on $\{1, \ldots, n\}$.

(2) The point $y_{i} \in N$ is a branch point whose preimages have multiplicities $\mu_{i 1}, \ldots, \mu_{i k_{i}}$, if and only if $\rho\left(\beta_{i}\right)$ is a nonidentity permutation with cycles of lengths $\mu_{i 1}+1, \ldots, \mu_{i k_{i}}+1$.

(3) If $N$ is nonorientable, then $M$ is nonorientable if and only if there exists a word $W(\alpha, \beta) \in \Pi$ with an odd number of $\alpha$ terms such that the permutation $W(\rho(\alpha), \rho(\beta))$ fixes $k$, for some $k \in\{1, \ldots, n\}$.

3. The classification problem. In this section we give necessary and sufficient conditions for an array $B$ to be the branching array for a degree $n$ branched cover onto any surface $N$ with $\chi(N) \leqslant 0$. Since the conditions are necessary for maps onto any surface but sufficient only for the ones with $\chi(N)<0$, we do the necessity and sufficiency parts in different theorems.

3.1. THEOREM. Let $f: M \rightarrow N$ be a degree $n$ branched cover between connected, closed surfaces with branch points $y_{1}, \ldots, y_{m}$ in $N$ where the multiplicities 
at points in $f^{-1}\left(y_{i}\right)$ are $\mu_{i 1}, \ldots, \mu_{i k_{i}}$. Then:

(1) $\Sigma\left\{\mu_{i j}+1 \mid j=1, \ldots, k_{i}\right\}=n$;

(2) $\mu=\Sigma\left\{\mu_{i j} \mid i=1, \ldots, m ; j=1, \ldots, k_{i}\right\}$ is an even number.

Furthermore, if $N$ is nonorientable and $n$ is odd, then $M$ is nonorientable.

Proof. (1) This condition is well known and can be shown directly from the definition of a branched cover. Note, however, that it follows immediately from 2.1(2) since the sum of the cycle lengths of a permutation in $S_{n}$ is $n$.

(2) Let $\rho$ be any homomorphism associated with $f$ by 2.1 and let $\Pi$ have the standard generators $\alpha_{i}, \beta_{j}$. We say the parity of a permutation is 0 if it is even and 1 if it is odd. Since each $\alpha_{i}, i=1, \ldots, r$, occurs twice in the word $W(\alpha)$, the parity of the permutation $W(\rho(\alpha))$ is 0 . Since $\rho$ is a homomorphism, $W(\rho(\beta)) W(\rho(\alpha))$ is the identity in $S_{n}$ and the parity of $W(\rho(\beta))$ is also 0 . By 2.1(2), the $j$ th cycle $c_{i j}$ in $\rho\left(\beta_{i}\right)$ has length $\mu_{i j}+1$, hence the parity of the cycle $c_{i j}, \operatorname{par}\left(c_{i j}\right)$, is congruent to $\mu_{i j}$ modulo 2. But $\operatorname{par}(W(\rho(\beta)))$ equals the mod 2 sum of the parities of all the cycles in $W(\rho(\beta))$, hence $0=\operatorname{par}(W(\rho(\beta))) \equiv$ $\mu(\bmod 2)$, and $\mu$ is even. Note that if $N$ is orientable, then this condition follows immediately from the Riemann-Hurwitz formula since the Euler characteristic of an orientable surface is even.

Now suppose $N$ is nonorientable and $n$ is odd. Then $\rho\left(\alpha_{1}\right)$ is a permutation of odd length and has at least one odd cycle, say $\left(c_{1} c_{2} \cdots c_{p}\right)$. Thus the word $\alpha_{1}^{p} \in \Pi$ has an odd number of occurrences of $\alpha$ terms and $\rho_{\alpha_{1}}^{p}\left(c_{1}\right)=c_{1}$. Thus $M$ is nonorientable by $2.1(3)$.

The following lemmas are used for the sufficiency part of the classification theorem. The first, Lemma 3.2, is done in [6]. For completeness, a proof is also given here.

\subsection{LEMMA. Every even permutation is the product of two cycles.}

Proof. Let $P$ be a given permutation and expand $P$ as a product of disjoint cycles labeling the first letter of each cycle as follows:

$$
P=\left(x_{1} X_{1}\right)\left(x_{2} X_{2}\right) \cdots\left(x_{j} X_{j}\right)\left(y_{1} Y_{1}\right)\left(y_{2} Y_{2}\right) \cdots\left(y_{k} Y_{k}\right),
$$

where the $X_{i}^{\prime}$ 's and $Y_{i}^{\prime}$ 's are (possibly empty) strings of the remaining letters in the cycles. The $x$ 's designate cycles of odd length and the $y$ 's cycles of even length. Let $X_{i}\left(Y_{i}\right)$ be the string consisting of the even terms (i.e. the 2nd, 4th, 6th, etc.) in $X_{i}\left(Y_{i}\right)$ and let $\bar{X}_{i}\left(\bar{Y}_{i}\right)$ be the string consisting of the odd terms. Let $Q$ be the cycle $\left(x_{1} \cdots x_{j} y_{1} \cdots y_{k} X_{1} \cdots X_{j} Y_{1} \cdots Y_{k}\right)$ and observe that the product $Q P$ (multiplying from the right) is the permutation

$$
\begin{aligned}
\left(x_{1} \underline{X}_{1} \cdots x_{j} \underline{X}_{j} y_{1} \underline{Y}_{1} \bar{Y}_{2} y_{3} \underline{Y}_{3} \bar{Y}_{4} y_{5} \cdots \bar{Y}_{k-1} y_{k} \underline{Y}_{k}\right) \\
\cdot\left(\bar{X}_{1} \cdots \bar{X}_{j} \bar{Y}_{1} y_{2} \underline{Y}_{2} \bar{Y}_{3} y_{4} \underline{Y}_{4} \bar{Y}_{5} \cdots \bar{Y}_{k}\right)
\end{aligned}
$$


for $k$ odd and

$$
\begin{aligned}
\left(x_{1} \underline{X}_{1} \cdots x_{j} \underline{X}_{j} y_{1} \underline{Y}_{1} \bar{Y}_{2} y_{3} \underline{Y}_{3} \bar{Y}_{4} y_{5} \cdots \bar{Y}_{k} \bar{X}_{1}\right. \\
\left.\cdots \bar{X}_{j} \bar{Y}_{1} y_{2} \underline{Y}_{2} \bar{Y}_{3} y_{4} \cdots \bar{Y}_{k-1} y_{k} \underline{Y}_{k}\right)
\end{aligned}
$$

for $k$ even. If $P$ is an even permutation then $k$ must be even and the product $Q P$ is a cycle. Hence $P=\left(Q^{-1}\right)(Q P)$ factors as claimed.

3.3. LEMMA. If $P$ and $Q$ are cyclic permutations, then there is a permutation $R$ having a cycle of length one such that $P=R^{-1} Q R$.

Proof. Let $P=\left(c_{1} c_{2} \ldots c_{n}\right)$ and $Q=\left(d_{1} d_{2} \ldots d_{n}\right)$. If $c_{i}=d_{j}$, take $R$ to be

$$
\left(\begin{array}{c}
c_{1} c_{2} \cdots c_{i} \cdots c_{n} \\
\cdots d_{j-1} d_{j} d_{j+1} \cdots
\end{array}\right)
$$

See $[3$, p. 55].

3.4. THEOREM. Let $N$ be any surface with $\chi(N) \leqslant 0$ and let $B$ be an array of nonnegative integers, $B=\left\{\mu_{i j} \mid i=1, \ldots, m ; j=1, \ldots, k_{i}\right\}$ (each row containing some nonzero entry) such that:

(1) $\Sigma\left\{\mu_{i j}+1 \mid j=1, \ldots, k_{i}\right\}=n$;

(2) $\mu=\Sigma\left\{\mu_{i j} \mid i=1, \ldots, m ; j=1, \ldots, k_{i}\right\}$ is an even number.

Then there exists a degree $n$ branched cover $f: M \rightarrow N$ from some connected surface $M$ with $B$ as its branching array. Furthermore, if $N$ is nonorientable and $n$ even, then $M$ can be chosen nonorientable.

Proof. The proof in the case where $N$ is orientable is done in [6]. Suppose $N$ is nonorientable and choose points $y_{1}, \ldots, y_{m}$ in $N$. Let $\alpha_{1}, \ldots, \alpha_{r}, \beta_{1}, \ldots, \beta_{m}$ be the generators of $\Pi$. We define a homomorphism $\rho: \Pi \rightarrow S_{n}$ by defining $\rho$ on the generators and extending to all of $\Pi$. Choose permutations $P_{\beta_{1}}, \ldots, P_{\beta_{m}}$ such that $P_{\beta_{i}}$ has cycles of lengths $\mu_{i 1}+1, \ldots, \mu_{i k_{i}}$ +1 . It follows from the fact that $\mu$ is even that the product $P_{\beta_{m}} \cdots P_{\beta_{1}}$ is an even permutation. Hence by 3.1 there is a permutation $Q$ such that $Q P_{\beta_{m}} \cdots P_{\beta_{1}}$ is a cycle. Since $Q^{-1}$ and $Q P_{\beta_{m}} \cdots P_{\beta_{1}}$ are cycles, they are conjugates and there is a permutation $P_{\alpha_{2}}$ such that $Q P_{\beta_{m}} \cdots P_{\beta_{1}}=$ $P_{\alpha_{2}} Q^{-1} P_{\alpha_{2}}^{-1}$; or equivalently,

$$
P_{\alpha_{2}} Q P_{\alpha_{2}}^{-1} Q P_{\beta_{m}} \cdots P_{\beta_{1}}=1 \text {. }
$$

Insert $P_{\alpha_{2}} P_{\alpha_{2}}^{-1}$ on the left to obtain

or equivalently,

$$
P_{\alpha_{2}} P_{\alpha_{2}} P_{\alpha_{2}}^{-1} Q P_{\alpha_{2}}^{-1} Q P_{\beta_{m}} \ldots P_{\beta_{1}}=1
$$

$$
\left(P_{\alpha_{2}}\right)^{2}\left(P_{\alpha_{2}}^{-1} Q\right)^{2} P_{\beta_{m}} \ldots P_{\beta_{1}}=1
$$

Let $P_{\alpha_{1}}=P_{\alpha_{2}}^{-1} Q$. Define $\rho\left(\beta_{i}\right)=P_{\beta_{i}}, i=1, \ldots, m, \rho\left(\alpha_{1}\right)=P_{\alpha_{1}}, \rho\left(\alpha_{2}\right)=P_{\alpha_{2}}$, 
and $\rho\left(\alpha_{i}\right)=1$, for $i=3, \ldots, r$. Hence $\rho^{2}\left(\alpha_{r}\right) \ldots \rho^{2}\left(\alpha_{1}\right) \rho\left(\beta_{m}\right) \ldots \rho\left(\beta_{1}\right)=1$ and $\rho$ extends to a homomorphism. Let $(f, M)$ be any branched covering space associated with $\rho$ by 2.1. Since $P_{\alpha_{1}} P_{\alpha_{2}}\left(=P_{\alpha_{2}}^{-1} Q P_{\alpha_{2}}\right)$ is a conjugate of $Q$ and hence a cycle, $\rho(\Pi)$ acts transitively on $\{1, \ldots, n\}$ and $M$ is connected by 2.1(1). The cover $f$ has $B$ as its branching array by 2.1(2) and the choice of the permutations $P_{\beta_{i}}, i=1, \ldots, m$. By 3.3, $P_{\alpha_{2}}$ can be chosen to have a cycle of length one. If this is done then $M$ is nonorientable by 2.1(3). (Take $W(\alpha, \beta)$ to be $\alpha_{2}$.)

The proof in the case where $N$ is orientable is similar. Choose $Q$ and the $P_{\beta_{1}}$ as before. Let $P_{\alpha_{2}}$ be the permutation such that $P_{\alpha_{2}}^{-1} Q P_{\alpha_{2}}=Q P_{\beta_{m}} \ldots P_{\beta_{1}}$. Take $P_{\alpha_{1}}=Q, P_{\alpha_{i}}=1$, for $i=3, \ldots, r$, and we have

$$
P_{\alpha_{r}}^{-1} P_{\alpha_{r-1}}^{-1} P_{\alpha_{r}} P_{\alpha_{r-1}} \ldots P_{\alpha_{2}}^{-1} P_{\alpha_{1}}^{-1} P_{\alpha_{2}} P_{\alpha_{1}} P_{\beta_{m}} \ldots P_{\beta_{1}}=1 \text {. }
$$

Now $\rho$ defined as before extends to a homomorphism.

We have shown that given an array $B$ satisfying conditions (1) and (2) above and a nonorientable surface $N$ (not the projective plane), there is always a degree $n$ branched cover having $B$ as its branching array. We observed that if $n$ is odd, the covering surface is always nonorientable, and if $n$ is even, there is always a nonorientable covering surface with the prescribed branching. However, if $n$ is even, there is not always an orientable covering surface with the prescribed branching. For example, there is no 4-1 orientable cover of the Klein bottle having one branch point with multiplicities of 0 and 2. If there were, there would be permutations $P_{\beta_{1}}, P_{\alpha_{1}}$, and $P_{\alpha_{2}}$ with $P_{\alpha_{2}}^{2} P_{\alpha_{1}}^{2} P_{\beta_{1}}$ $=1, P_{\beta_{1}}$ having one cycle of length one and one of length three, and the permutations $P_{\alpha_{1}}$ and $P_{\alpha_{2}}$ having no odd cycles. But no such permutations exist in $S_{4}$.

Finally, we note that if $N$ is the sphere $S^{2}$ or the projective plane $P^{2}$, then the Riemann-Hurwitz formula places a third restriction on branching arrays of maps onto $N$; namely,

(3) $\mu>2 n-2$, for $N=S^{2}$;

(3) $\mu \geqslant n-2$, for $N=P^{2}$.

However, these conditions combined with (1) and (2) of 3.4 are not enough to characterize the branching arrays of maps onto $S^{2}$ and $P^{2}$. This is shown in the following examples, the first of which appears in [6].

3.5. EXAMPLE. The array

$$
B=\left[\begin{array}{ll}
1 & 1 \\
1 & 1 \\
0 & 2
\end{array}\right]
$$

satisfies conditions (1), (2), and (3), but there is no degree 4 branched cover onto $S^{2}$ with branching array $B$. If there were, there would be permutations $P_{\beta_{1}}, P_{\beta_{2}}$, and $P_{\beta_{3}}$ in $S_{4}$ with $P_{\beta_{1}}$ and $P_{\beta_{2}}$ having two 2-cycles and $P_{\beta_{3}}$ having a 
1-cycle and a 3-cycle such that $P_{\beta_{3}} P_{\beta_{2}} P_{\beta_{1}}=1$. But no such permutations exist in $S_{4}$.

3.6. EXAMPLE. The array $B=\left[\begin{array}{ll}1 & 3\end{array}\right]$ satisfies conditions (1), (2), and (3), but there is no 6-1 branched cover of $P^{2}$ with branching array $B$. If there were, there would be permutations $P_{\beta_{1}}$ and $P_{\alpha_{1}}$ in $S_{6}$ with $P_{\beta_{1}}$ having a 2-cycle and a 4-cycle such that $P_{\alpha_{1}} P_{\beta_{1}}=1$. But there are no such permutations in $S_{6}$.

\section{REFERENCES}

1. O. Endler, Compact Riemann surfaces with prescribed ramifications and Puiseux series, Bol. Soc. Brasil. Mat. 2 (1971), 61-64.

2. G. Francis, Assembling Riemann surfaces with given boundary curves and branch points, Illinois J. Math. 20 (1976), 198-217.

3. M. Hall, The theory of groups, Macmillan, New York, 1959.

4. A. Hurwitz, Riemann'sche Flächen mit gegebenen Verzweigungspunkten, Math. Ann. 39 (1891), 1-61.

5. W. S. Massey, Algebraic topology: An introduction, Harcourt, Brace, and World, New York, 1967.

6. C. B. Shepardson, Hurwitz-Riemann formulas, Ph.D. thesis, Syracuse Univ., 1973.

7. R. Thom, L'équivalence d'une fonction différentiable et d'une polynôme, Topology 3 (1965), suppl. 2, 297-307.

Department of Mathematics, Vanderbitt University, Nashville, Tennessee 37235

Current address: Department of Mathematics, Stetson University, DeLand, Florida 32720 